Simopoulos AP, Ordovas JM (eds): Nutrigenetics and Nutrigenomics.

World Rev Nutr Diet. Basel, Karger, 2004, vol 93, pp 303-311

\title{
Appendix I
}

\section{Glossary of Genetic Terms}

Allele - Alternative form of a genetic locus; a single allele for each locus is inherited from each parent (e.g. at a locus for eye color the allele might result in blue or brown eyes).

Apoptosis - Programmed cell death, the body's normal method of disposing of damaged, unwanted, or unneeded cells.

Arrayed library - Individual primary recombinant clones (hosted in phage, cosmid, YAC, or other vector) that are placed in two-dimensional arrays in microtiter dishes. Each primary clone can be identified by the identity of the plate and the clone location (row and column) on that plate. Arrayed libraries of clones can be used for many applications, including screening for a specific gene or genomic region of interest.

Autosomal dominant - A gene on one of the non-sex chromosomes that is always expressed, even if only one copy is present. The chance of passing the gene to offspring is $50 \%$ for each pregnancy.

Autosome - A chromosome not involved in sex determination. The diploid human genome consists of a total of 46 chromosomes: 22 pairs of autosomes, and 1 pair of sex chromosomes (the $\mathrm{X}$ and $\mathrm{Y}$ chromosomes).

Basepair (bp) - Two nitrogenous bases (adenine and thymine or guanine and cytosine) held together by weak bonds. Two strands of DNA are held together in the shape of a double helix by the bonds between base pairs.

Base sequence - The order of nucleotide bases in a DNA molecule; determines structure of proteins encoded by that DNA.

Base sequence analysis - A method, sometimes automated, for determining the base sequence.

Bioinformatics or computational biology - The science of managing and analyzing biological data using advanced computing techniques. Especially important in analyzing genomic research data. 
Biotechnology - A set of biological techniques developed through basic research and now applied to research and product development. In particular, biotechnology refers to the use by industry of recombinant DNA, cell fusion, and new bioprocessing techniques.

Candidate gene - A gene located in a chromosome region suspected of being involved in a disease.

Capillary array - Gel-filled silica capillaries used to separate fragments or DNA sequencing. The small diameter of the capillaries permits the application of higher electric fields, providing high speed, high throughput separations that are significantly faster than traditional slab gels.

Carrier - An individual who possesses an unexpressed, recessive trait.

cDNA library - A collection of DNA sequences that code for genes. The sequences are generated in the laboratory from mRNA sequences.

Centimorgan (cM) - A unit of measure of recombination frequency. One centimorgan is equal to a $1 \%$ chance that a marker at one genetic locus will be separated from a marker at a second locus due to crossing over in a single generation. In human beings, one centimorgan is equivalent, on average, to one million basepairs.

Clone - An exact copy made of biological material such as a DNA segment (e.g. a gene or other region), a whole cell, or a complete organism.

Comparative genomics - The study of human genetics by comparisons with model organisms such as mice, the fruit fly, and the bacterium Escherichia coli.

Complementary DNA (cDNA) - DNA that is synthesized in the laboratory from a messenger RNA template.

Complementary sequence - Nucleic acid base sequence that can form a double-stranded structure with another DNA fragment by following basepairing rules (A pairs with $\mathrm{T}$ and $\mathrm{C}$ with $\mathrm{G}$ ). The complementary sequence to GTAC for example, is CATG.

Complex trait - Trait that has a genetic component that does not follow strict Mendelian inheritance. May involve the interaction of two or more genes or gene-environment interactions.

Conserved sequence - A base sequence in a DNA molecule (or an amino acid sequence in a protein) that has remained essentially unchanged throughout evolution.

Deletion - A loss of part of the DNA from a chromosome; can lead to a disease or abnormality.

Deletion map - A description of a specific chromosome that uses defined mutations - specific deleted areas in the genome - as 'biochemical signposts,' or markers for specific areas.

Disease-associated genes - Alleles carrying particular DNA sequences associated with the presence of disease. 
DNA (deoxyribonucleic acid) - The molecule that encodes genetic information. DNA is a double-stranded molecule held together by weak bonds between base pairs of nucleotides. The four nucleotides in DNA contain the bases adenine $(A)$, guanine $(\mathrm{G})$, cytosine $(\mathrm{C})$, and thymine $(\mathrm{T})$. In nature, base pairs form only between $A$ and $T$ and between $G$ and $C$; thus the base sequence of each single strand can be deduced from that of its partner.

DNA repair genes - Genes encoding proteins that correct errors in DNA sequencing.

Epistasis - One gene interferes with or prevents the expression of another gene located at a different locus.

Exon - The protein-coding DNA sequence of a gene.

Fingerprinting - In genetics, the identification of multiple specific alleles on a person's DNA to produce a unique identifier for that person.

Functional genomics - The study of genes, their resulting proteins, and the role played by the proteins in the body's biochemical processes.

GC-rich area - Many DNA sequences carry long stretches of repeated G and $\mathrm{C}$ which often indicate a gene-rich region.

Gene - The fundamental physical and functional unit of heredity. A gene is an ordered sequence of nucleotides located in a particular position on a particular chromosome that encodes a specific functional product (i.e. a protein or RNA molecule).

Gene chip technology - Development of cDNA microarrays from a large number of genes. Used to monitor and measure changes in gene expression for each gene represented on the chip.

Gene expression - The process by which a gene's coded information is converted into the structures present and operating in the cell. Expressed genes include those that are transcribed into mRNA and then translated into protein and those that are transcribed into RNA but not translated into protein (e.g. transfer and ribosomal RNAs).

Gene family - Group of closely related genes that make similar products.

Gene mapping - Determination of the relative positions of genes on a DNA molecule (chromosome or plasmid) and of the distance, in linkage units or physical units, between them.

Gene pool - All the variations of genes in a species.

Gene prediction - Predictions of possible genes made by a computer program based on how well a stretch of DNA sequence matches known gene sequences.

Gene product - The biochemical material, either RNA or protein, resulting from expression of a gene. The amount of gene product is used to measure how active a gene is; abnormal amounts can be correlated with disease-causing alleles. 
Gene therapy - An experimental procedure aimed at replacing, manipulating, or supplementing nonfunctional or misfunctioning genes with healthy genes.

Gene transfer - Incorporation of new DNA into an organism's cells, usually by a vector such as a modified virus. Used in gene therapy.

Genetic code - The sequence of nucleotides, coded in triplets (codons) along the mRNA, that determines the sequence of amino acids in protein synthesis. A gene's DNA sequence can be used to predict the mRNA sequence, and the genetic code can in turn be used to predict the amino acid sequence.

Genetic counseling - Provides patients and their families with education and information about genetic-related conditions and helps them make informed decisions.

Genetic engineering - Altering the genetic material of cells or organisms to enable them to make new substances or perform new functions.

Genetic illness - Sickness, physical disability, or other disorder resulting from the inheritance of one or more deleterious alleles.

Genetic marker - A gene or other identifiable portion of DNA whose inheritance can be followed.

Genetic polymorphism - Difference in DNA sequence among individuals, groups, or populations (e.g. genes for blue eyes versus brown eyes).

Genetic predisposition - Susceptibility to a genetic disease. May or may not result in actual development of the disease.

Genetic screening - Testing a group of people to identify individuals at high risk of having or passing on a specific genetic disorder.

Genetic testing - Analyzing an individual's genetic material to determine predisposition to a particular health condition or to confirm a diagnosis of genetic disease.

Genetics - The study of inheritance patterns of specific traits.

Genome - All the genetic material in the chromosomes of a particular organism; its size is generally given as its total number of basepairs.

Genome project - Research and technology-development effort aimed at mapping and sequencing the genome of human beings and certain model organisms.

Genomic library - A collection of clones made from a set of randomly generated overlapping DNA fragments that represent the entire genome of an organism.

Genomics - The study of genes and their function.

Genotype - The genetic constitution of an organism, as distinguished from its physical appearance (its phenotype).

Germ cell - Sperm and egg cells and their precursors. Germ cells are haploid and have only one set of chromosomes ( 23 in all), while all other cells have two copies (46 in all). 
Haplotype - A way of denoting the collective genotype of a number of closely linked loci on a chromosome.

High-throughput sequencing - A fast method of determining the order of bases in DNA.

Imprinting - A phenomenon in which the disease phenotype depends on which parent passed on the disease gene. For instance, both Prader-Willi and Angelman syndromes are inherited when the same part of chromosome 15 is missing. When the father's complement of 15 is missing, the child has PraderWilli, but when the mother's complement of 15 is missing, the child has Angelman syndrome.

Intron - DNA sequence that interrupts the protein-coding sequence of a gene; an intron is transcribed into RNA but is cut out of the message before it is translated into protein.

Kilobase (kb) - Unit of length for DNA fragments equal to 1,000 nucleotides.

Knockout - Deactivation of specific genes; used in laboratory organisms to study gene function.

Linkage - The proximity of two or more markers (e.g. genes, RFLP markers) on a chromosome; the closer the markers, the lower the probability that they will be separated during DNA repair or replication processes (binary fission in prokaryotes, mitosis or meiosis in eukaryotes), and hence the greater the probability that they will be inherited together.

Linkage disequilibrium - Where alleles occur together more often than can be accounted for by chance. Indicates that the two alleles are physically close on the DNA strand.

Linkage map - A map of the relative positions of genetic loci on a chromosome, determined on the basis of how often the loci are inherited together. Distance is measured in centimorgans (cM).

Locus (pl. loci) - The position on a chromosome of a gene or other chromosome marker; also, the DNA at that position. The use of locus is sometimes restricted to mean expressed DNA regions.

Megabase (Mb) - Unit of length for DNA fragments equal to 1 million nucleotides and roughly equal to $1 \mathrm{cM}$.

Messenger RNA (mRNA) - RNA that serves as a template for protein synthesis.

Metabolome - The quantitative complement of all the low molecular weight molecules present in cells in a particular physiological or developmental state.

Metabonomics and Metabolomics - These very similar terms have arisen at about the same time in different area of bioscience research, mainly animal biochemistry and microbial/plant biochemistry respectively. Although both involve the multiparametric measurement of metabolites they are not philosophically identical as metabonomics deals with integrated, multicellular, 
biological systems including communicating extracellular environments and metabolomics deals with simple cell systems and, at least in terms of published data, mainly intracellular metabolite concentrations.

Microarray - Sets of miniaturized chemical reaction areas that may also be used to test DNA fragments, antibodies, or proteins.

Mitochondrial DNA - The genetic material found in mitochondria, the organelles that generate energy for the cell. Not inherited in the same fashion as nucleic DNA.

Molecular genetics - The study of macromolecules important in biological inheritance.

Molecular medicine - The treatment of injury or disease at the molecular level. Examples include the use of DNA-based diagnostic tests or medicine derived from DNA sequence information.

Multifactorial or multigenic disorder - See polygenic disorder.

Mutation - Any heritable change in DNA sequence.

Nutrigenetics - Refers to an individual's specific response to diet due to genetic variants or polymorphisms (i.e. individuals responding differently to the same diet by having different levels of, for example, serum cholesterol and blood pressure because of genetic variation).

Nutrigenomics - Refers to the role of nutrients in gene expression (i.e. polyunsaturated fatty acids suppress fatty acid synthase (mRNA) gene expression).

Oligogenic - A phenotypic trait produced by two or more genes working together.

Pharmacogenomics - The study of the interaction of an individual's genetic makeup and response to a drug.

Phenotype - The physical characteristics of an organism or the presence of a disease that may or may not be genetic.

Physical map - A map of the locations of identifiable landmarks on DNA (e.g. restriction-enzyme cutting sites, genes), regardless of inheritance. Distance is measured in base pairs. For the human genome, the lowest-resolution physical map is the banding patterns on the 24 different chromosomes; the highestresolution map is the complete nucleotide sequence of the chromosomes.

Polygenic disorder - Genetic disorder resulting from the combined action of alleles of more than one gene (e.g. heart disease, diabetes, and some cancers). Although such disorders are inherited, they depend on the simultaneous presence of several alleles; thus, the hereditary patterns usually are more complex than those of single-gene disorders.

Polymorphism - Difference in DNA sequence among individuals that may underlie differences in health. Genetic variations occurring in more than $1 \%$ of a population would be considered useful polymorphisms for genetic linkage analysis. 
Population genetics - The study of variation in genes among a group of individuals.

Positional cloning - A technique used to identify genes, usually those that are associated with diseases, based on their location on a chromosome.

Privacy - In genetics, the right of people to restrict access to their genetic information.

Probe - Single-stranded DNA or RNA molecules of specific base sequence, labeled either radioactively or immunologically, that are used to detect the complementary base sequence by hybridization.

Prokaryote - Cell or organism lacking a membrane-bound, structurally discrete nucleus and other subcellular compartments. Bacteria are examples of prokaryotes.

Promoter - A DNA site to which RNA polymerase will bind and initiate transcription.

Proteome - Proteins expressed by a cell or organ at a particular time and under specific conditions.

Proteomics - The study of the full set of proteins encoded by a genome. The measurement of 'all' cellular protein production and levels, the structural characterization of those proteins and the understanding of their functions. This science is also heavily dependent on advanced analytical methodologies, including for example 2D gel-electrophoresis combined with nanospray mass spectrometry for separation and identification of proteins. Interestingly in humans, there may be only about 30,000 genes, but there are thought to be many more cellular proteins than there are genes, including all the possible post-translational modifications. This poses an immediate theoretical problem when gene expression-proteomic correlations are being sought as there is a higher level of cellular control than the genome which is in the protein complement itself. Also changes in gene expression which may or may not result in changes in cellular protein synthesis have to occur at different times in the cell, and different gene regulation events occurring at the same time may take different times to effect the proteome. From an analytical viewpoint, so far it has only been possible to separate and identify a small fraction of the possible cellular proteins.

Pseudogene - A sequence of DNA similar to a gene but nonfunctional; probably the remnant of a once-functional gene that accumulated mutations.

Recombinant DNA technology - Procedure used to join together DNA segments in a cell-free system (an environment outside a cell or organism). Under appropriate conditions, a recombinant DNA molecule can enter a cell and replicate there, either autonomously or after it has become integrated into a cellular chromosome. 
Recombination - The process by which progeny derive a combination of genes different from that of either parent. In higher organisms, this can occur by crossing over.

Regulatory region or sequence - A DNA base sequence that controls gene expression.

Repetitive DNA - Sequences of varying lengths that occur in multiple copies in the genome; it represents much of the human genome.

Restriction fragment length polymorphism (RFLP) - Variation between individuals in DNA fragment sizes cut by specific restriction enzymes; polymorphic sequences that result in RFLPs are used as markers on both physical maps and genetic linkage maps. RFLPs usually are caused by mutation at a cutting site.

Risk communication - In genetics, a process in which a genetic counselor or other medical professional interprets genetic results and advises patients of the consequences for them and their offspring.

RNA (ribonucleic acid) - A chemical found in the nucleus and cytoplasm of cells; it plays an important role in protein synthesis and other chemical activities of the cell. The structure of RNA is similar to that of DNA. There are several classes of RNA molecules, including messenger RNA, transfer RNA, ribosomal RNA, and other small RNAs, each serving a different purpose.

Sequence tagged site (STS) - Short (200 to 500 basepairs) DNA sequence that has a single occurrence in the human genome and whose location and base sequence are known. Detectable by polymerase chain reaction, STSs are useful for localizing and orienting the mapping and sequence data reported from many different laboratories and serve as landmarks on the developing physical map of the human genome. Expressed sequence tags (ESTs) are STSs derived from cDNAs.

Sequencing - Determination of the order of nucleotides (base sequences) in a DNA or RNA molecule or the order of amino acids in a protein.

Sequencing technology - The instrumentation and procedures used to determine the order of nucleotides in DNA.

Single nucleotide polymorphism (SNP) - DNA sequence variations that occur when a single nucleotide (A, T, C, or G) in the genome sequence is altered.

Structural genomics - The effort to determine the 3D structures of large numbers of proteins using both experimental techniques and computer simulation.

Toxicogenomics - The study of how genomes respond to environmental stressors or toxicants. Combines genome-wide mRNA expression profiling with protein expression patterns using bioinformatics to understand the role of gene-environment interactions in disease and dysfunction.

Transcription - The synthesis of an RNA copy from a sequence of DNA (a gene); the first step in gene expression. 
Transcriptome - The full complement of activated genes, mRNAs or transcripts in a particular tissue at a particular time.

or

Transcriptome - The transcriptome can be defined as the complete collection of transcribed elements of the genome. In addition to mRNAs, it also represents non-coding RNAs, which are used for structural and regulatory purposes. Alterations in the structure or levels of expression of any one of these RNAs or their proteins can contribute to disease. An understanding of the transcriptome will provide a valuable tool in the research for novel drugs.

Transcriptomics - This is the quantitative measurement of gene expression in a cell or tissue. Generally this involves the measurement of mRNA levels by various methods, the most popular currently being via proprietary gene chips. The problems here include the fact that chips are very expensive, that many genes or sequences have no known function and that the relationships between quantitative variation or patterns in expression and the influences of cell or pathway function are, at best, poorly understood. Moreover, it is widely appreciated that mRNAs are not chemically stable and steps must be taken to ensure quantitative reliability of the chip measurements. A less well considered problem stems from the fact that quite large samples of tissue are generally required to make an extensive set of gene expression measurements on one sample (up to $1 \mathrm{~g}$ in the case of human tissues). In such sample, even in a relatively 'homogeneous' tissue such as liver, there may be dozens of cell types in different topographical locations performing different functions and by definition have different levels of genetic activity. The gene chip measures an average of these activities the meaning of which is unclear.

Transfection - The introduction of foreign DNA into a host cell.

Transfer RNA (tRNA) - A class of RNA having structures with triplet nucleotide sequences that are complementary to the triplet nucleotide coding sequences of mRNA. The role of tRNAs in protein synthesis is to bond with amino acids and transfer them to the ribosomes, where proteins are assembled according to the genetic code carried by mRNA.

Transformation - A process by which the genetic material carried by an individual cell is altered by incorporation of exogenous DNA into its genome.

Transgenic - An experimentally produced organism in which DNA has been artificially introduced and incorporated into the organism's germ line.

Translation - The process in which the genetic code carried by mRNA directs the synthesis of proteins from amino acids.

Wild type - The form of an organism that occurs most frequently in nature.

Yeast artificial chromosome (YAC) - Constructed from yeast DNA, it is a vector used to clone large DNA fragments. 